\title{
Filosofía moral, cultura y educación. Aportes para un diseño curricular actual ${ }^{1}$
}

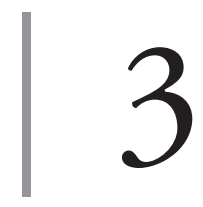

\author{
https://doi.org/10.21830/9789585377127.03
}

Luis Carlos Pérez Ferro ${ }^{2}$

Escuela Militar de Cadetes "General José María Córdova”

\section{Resumen}

Este capítulo de libro presenta un rastreo teórico reflexivo sobre los conceptos de hombre como fin, reconocimiento del imperativo categórico y autonomía de la voluntad en Kant, expresados como principios de la formación curricular en ética (filosofía moral) y en consideración a la cultura y sus definiciones. Es un ejercicio de construcción teorético y se sirve de elementos propios de la indagación reflexiva documental. Se concluye que la formación curricular debe ser un proceso caracterizado por el diálogo filosófico en todos los niveles de formación, de tal manera que involucre las dimensiones macro, meso y microcurricular, así como la búsqueda de estrategias innovadoras que faciliten la reflexión de las acciones morales en uso de la razón, y de decisiones personales validadas por su posibilidad de considerarse aplicables universalmente.

Palabras clave: cultura; currículo; educación; ética; filosofía; Kant.

1 Este capítulo presenta los resultados colaborativos de dos proyectos de investigación: (1) "Ética militar en entornos complejos de seguridad y defensa: lecturas y aportes desde la experiencia de las Fuerzas Militares de Colombia”, del grupo de investigación Masa Crítica, de la Escuela Superior de Guerra "General Rafael Reyes Prieto", Colombia, categorizado en B por Minciencias y con código de registro COL0123247, y (2) "Ética y corrupción", del grupo de investigación en Ciencias Militares de la Escuela Militar de Cadetes "General José María Córdova”, Colombia, categorizado en B por Minciencias y con código de registro COL0082556. Los puntos de vista pertenecen al autor y no reflejan necesariamente los de las instituciones participantes.

2 Magíster en Educación de la Pontificia Universidad Javeriana, Bogotá, Colombia. Psicólogo de la Universidad Santo Tomás, Colombia. Estudios en Filosofía en el Seminario Mayor Arquidiócesis de Bogotá. Profesor asociado de Ética de la Escuela Militar de Cadetes "General José María Córdova”, Colombia. Orcid: https://orcid.org/0000-0002-4793-7082 - Contacto: luisperezferro@cedoc.edu.co 


\section{Introducción}

La complejidad "en tanto problema, conjunto de teorías y métodos y, en un sentido más general, como campo de estudios de la ciencia contemporánea, o mejor aún, como un paradigma científico emergente" (Rodríguez et al., 2011, p. 5) ha puesto en escena reflexiones importantes sobre cómo han de ser comprendidas las acciones éticas. Esto, de profunda importancia en la búsqueda de sentido actual, ha vinculado a la filosofía moral a diversas actividades sociales, entre las cuales se destaca acá al fenómeno educativo. Pero, ¿cómo han de ser las relaciones entre ética y educación? ¿Es posible establecer estas relaciones? Lo anterior constituye un punto de partida para poder interrogarse sobre la formación ética dentro del ámbito educativo.

Así, este capítulo explora los atributos de la filosofía moral en la comprensión que Kant (1785) formula en su obra Fundamentación de una metafísica de las costumbres. Luego, se enuncian algunos elementos sobre el concepto de cultura para establecer sus vínculos. Finalmente, se indican algunas consideraciones en lo que se propone denominar cultura de la formación ética.

Con este propósito, se propone que, desde el punto de vista de la filosofía moral kantiana, la consideración del hombre como finalidad, la presencia del imperativo categórico y el desarrollo de la autonomía de la voluntad constituyen tres ejes estructurales que es posible expresar en el diseño y funcionamiento curricular de la formación ética en el ámbito de la educación. Quiere decir esto, que los derroteros que sirven como pivote para la formación ética amplia deberán, de alguna u otra forma, tener en consideración estos tres aspectos. Este trabajo, entonces, busca la construcción de puentes discursivos entre los conceptos kantianos indicados, la cultura y la educación.

\section{Marco teórico}

\section{Sobre la ética de las costumbres}

Empecemos por señalar que, dentro de toda la estructura de la ética en Kant, y particularmente en el prólogo de la Fundamentación, se presentan dos tipos de conocimiento: el de la razón, que es de naturaleza formal, referido a las formas del entendimiento desde la lógica, y el conocimiento de la razón 
material, circunscrito a los objetos. Este, a su vez, puede referirse a las leyes de la naturaleza y a las leyes de la libertad. Las primeras tratan sobre la física, en donde se analiza una filosofía de la naturaleza; allí, “todo sucede”. Las leyes de la libertad se refieren a un conocimiento ético o filosofía de la moral, cuyo análisis se centra en la voluntad humana, es decir, sobre todo lo que debe suceder.

Respecto a lo anterior, Kant indica que

cualquier conocimiento de la razón es material, y considera a algún objeto, o formal, y se ocupa simplemente de la forma del entendimiento y de la propia razón, así como en las reglas universales del pensar en general, sin distinguir entre los objetos. La filosofía formal se llama lógica, mientras que la material, la cual trata con determinados objetos y las leyes a que se hayan sometidos estos, se divide a su vez en dos. Pues esas leyes lo son de la naturaleza o de la libertad. La ciencia que versa sobre las primeras recibe el nombre de física y la que versa sobre las segundas el de ética; aquella se denomina también teoría de la naturaleza y esta teoría de las costumbres. (Kant 1785, p. 67)

Agrega, más adelante, que toda filosofía es empírica, en cuanto esta se sustenta sobre fundamentos de la experiencia, pero se diferencia de la filosofía pura, dado que esta se centra en teorías que parten exclusivamente de principios a priori. Esta última, cuando es meramente formal, se llama lógica, pero si se circunscribe a determinados objetos del entendimiento recibe el nombre de metafísica. Nace así la idea de una doble metafísica: una metafísica de la naturaleza y una metafísica de las costumbres. Luego, concluye que la ética tendrá una parte empírica, pero también una racional, la primera se denomina antropología práctica, y la puramente racional puede ser llamada con toda propiedad moral.

Lo anterior sirve de escenario epistémico y ético en la comprensión de los principios que estructuran la teoría de la moralidad en Kant. Por supuesto, acá se plantean de manera amplia y con un carácter sucinto; son útiles en la medida en que proveen principios generales para comprender el punto de partida de lo que a continuación se expone.

Como ya se mencionó en las primeras líneas, el desarrollo de la humanidad y el nivel de comprensión que tenemos en la actualidad de su constitución y funcionamiento incluyen los análisis sobre la conducta moral. Sin embargo, 
es evidente que, en distintas interacciones humanas, exista un nivel más o menos importante a considerar al otro como un instrumento para el logro de objetivos personales. Esto se debe posiblemente a diversas razones de orden cultural, que son imperantes en nuestro momento histórico específico. Sin pretender hacer un juzgamiento de estas condiciones, la actualidad y la forma en la cual nos relacionamos presta notable interés a la realización personal por encima de la realización ajena, en cambio de obrar tanto en los idearios personales y simultáneamente en los de los demás. ¿Qué implica entender o valorar lo que está fuera de mí, al otro, como instrumento y no como fin? Es este asunto, precisamente, uno de los elementos expuestos por Kant y que ahora se pone de relieve, en primer lugar, como eje de formación en la cultura de la ética. Luego, se analiza la formación en el reconocimiento de imperativos categóricos y, por último, se comenta la autonomía de la voluntad.

La tradición filosófica entera y particularmente la filosofía de la moral ha prestado interés al escrutinio de los componentes de la estructura del pensamiento racional, pero es precisamente Kant quien, ya en la modernidad, indica la perentoria necesidad de comprender a las personas como un valor absoluto, fines en sí mismos, y no instrumentos para el logro del avance individual y satisfacción de necesidades particulares.

Esto, señalado por el filósofo, es de vital importancia en la construcción o planeación de los currículos en ética. Comporta la necesidad de hacer un reconocimiento de los entramados sociales actuales, un diagnóstico de la calidad de las relaciones que viven hoy los estudiantes, mediadas por las influencias culturales más relevantes. La conciencia de las tendencias históricas diversas apuntan desde variadas miradas a poner en primer lugar los intereses o propensiones personales, y llamar a los otros como fuentes o vías para esos objetivos. Es apenas comprensible que esto puede traer beneficios pasajeros, pero a la postre, en el futuro próximo acarreará situaciones de frustración, soledad e individualismo. Esta condición de potenciación de los propios y detrimento de lo ajeno, directa o indirectamente, se proyecta en las condiciones actuales en las cuales viven más de un tercio de la población mundial.

En consecuencia, diseñar propuestas curriculares que conciban al hombre como finalidad en sí mismo constituye un avance significativo en la educación, la humanización y el desarrollo global. Pero, ¿cómo construir tales propuestas? En primer lugar, recordemos a Kant: 
El hombre y en general todo ser racional existe como fin en sí mismo, no simplemente como un medio para ser utilizado discrecionalmente por esta o aquella voluntad, sino que tanto en las acciones orientadas hacia sí mismo como en las dirigidas hacia otros seres racionales el hombre ha de ser considerado siempre al mismo tiempo como un fin [...] las personas, por lo tanto, no son meros fines subjetivos cuya existencia tiene un valor para nosotros como efecto de nuestra acción, sino que constituyen fines objetivos, es decir, cosas cuya existencia supone un fin en sí mismo y, a decir verdad, un fin tal, en cuyo lugar no puede ser colocado en ningún otro fin, al servicio del cual debiera quedar aquel simplemente como medio. (Kant, 1785, p. 138)

Globalmente, la máxima que ubica al hombre como finalidad y valor absoluto, hacedor constitutivo del reino de los fines (expresión utilizada por Kant), es un principio esencial en la planeación de cualquier formación en ética. Significa, entonces, admitir en el obrar propio la presencia del otro. Esto implica que la evaluación de mis acciones y su cumplimiento de bondad moral supone que debe ser, al mismo tiempo, universalmente aplicable a todos. En otras palabras, y de manera simple, la formación debe proveer al sujeto de competencias para reconocer y evaluar sus acciones como si ellas fuesen recibidas de parte de otros. Obra según tu criterio, de forma tal que lo que hagas pueda ser aplicado como principio universal de actuación.

Pero, ¿cómo lograr ese estado?, ¿cómo poder comprender racionalmente mis acciones y su validez universal? Es decir, actúa con los otros como quisieras ser tratado tú. En principio, la exploración de este interrogante nuevamente tiene respuesta en la argumentación kantiana, en el concepto de imperativo categórico. A continuación, se desarrollan algunas ideas que ubican este aspecto como máxima o principio básico en la planeación de cualquier currículo y acción educativa que tenga la ética como centro.

El concepto de imperativo categórico en Kant ha sido usado de diversas maneras y cuenta con interpretaciones desde corrientes no solo filosóficas, sino, en general, en las ciencias sociales. El imperativo se asume como una prescripción apremiante de conducta, de obrar, de actuación, y es categórico en la medida en que se manifiesta con una finalidad que no admite condición, es decir, que no está mediada por la instrumentalización de los deseos ni las necesidades humanas. Sobre el particular, Kant indica que 
el imperativo categórico sería el que representaría una acción como objetivamente necesaria por sí misma, sin referencia a ningún otro fin. Como toda ley práctica representa una acción posible como buena y, por ello como necesaria para un sujeto susceptible de verse determinado prácticamente por la razón, todos los imperativos constituyen fórmulas para determinar la acción que es necesaria según el principio de una voluntad buena de uno u otro modo. $\mathrm{Si}$ la acción fuese simplemente buena como medio para otra cosa, entonces el imperativo es hipotético; si se representa como buena en sí, o sea, como necesaria en una voluntad conforme de suyo con la razón, es categórico. (Kant, 1785, p. 114)

Así, tanto el imperativo categórico, como el presupuesto de entender lo humano como finalidad y no como instrumento presentan hasta acá dos de los elementos centrales que se proponen como fuente de cualquier currículo en ética. El reconocimiento de los imperativos categóricos en uso de la acción moral es una tarea ardua dentro de las acciones educativas; entonces, ¿qué implica educar en moralidad?, ¿qué implica formar deberes categóricos? Esto supone la construcción, planeación y gestión de contenidos curriculares que lleven a la reflexión y al reconocimiento de la moral como una estructura inalienable del ser humano. La moral como estructura implica la imposibilidad de no decidir continuamente. Dado que vivimos en un mundo donde las decisiones que se toman son el plato del día, es imposible no decidir, en consecuencia, la acción moral, el uso de los mandatos categóricos, es ineluctable.

Incidentalmente, la toma de decisiones, su entrenamiento y la valoración de las implicaciones morales, en uso siempre de los mandatos categóricos, ha de ser la estrategia preferida por las acciones educativas cuyo centro gravite en la ética. Sin embargo, estos procesos educacionales relacionados con las decisiones morales habrán de ser implementados en uso de uno de los principios categóricos centrales en el discurso kantiano: obra con la máxima que pueda hacer de si al mismo tiempo, una ley universal, o, en otras palabras, obra según una máxima que contenga dentro de si a la vez su propia validez universal para todo ser racional. Implica que todo ser racional, como fin en sí mismo, habría de poder considerarse al mismo tiempo como legislador universal con respecto a todas las leyes a las que pueda verse sometido. 
En tercer lugar, se propone acá la indagación de la autonomía de la voluntad como propósito educativo y línea argumental del presente artículo de reflexión. Sobre el particular, anota Kant:

Cada cosa de la naturaleza opera con arreglo a leyes. Solo un ser racional posee la capacidad de obrar según la representación de las leyes o con arreglo a principios del obrar, esto es, posee una voluntad. Como para derivar las acciones a partir de leyes se requiere una razón, la voluntad no es otra cosa que razón práctica. (Kant, 1785, p. 111)

Pero, ¿qué significa razón práctica?, ¿̇es susceptible de ser educada? Estas preguntas que marcan una línea de discusión indican que no es posible educar en principios éticos si no se fortalece el ejercicio de la voluntad en arreglo de la autonomía. En coherencia, habrán de diseñarse y desarrollarse estrategias educativas que fortalezcan el reconocimiento de las leyes universales que constituyen los principios morales, además de facilitar la introspección del sujeto para, posibilitar de este resultado, un obrar que atienda las leyes universales, dadas de manera a priori, y consecuentemente revertirlas a través del juicio racional y voluntario, al mundo propio y auténtico de la persona.

\section{La cultura y sus miradas. Rastreo de los trazos más representativos en la organización de su concepto}

La plasticidad del concepto de cultura implica la convergencia de diferentes miradas y sentidos, complejidad que se incrementa cuando se traza la tarea de indagar sobre sus atributos fundamentales, además de proponer consideraciones de interpretación sobe cada uno de ellos.

La persistente ambigüedad del concepto de cultura es notoria. Lo es mucho menos la idea de que esta ambigüedad no se deriva tanto de la forma en que la gente define la cultura como de la incompatibilidad existente entre numerosas líneas de pensamiento que han convergido históricamente sobre el mismo término. (Bauman, 2010, p. 95)

En el estudio de la cultura, su caracterización, estructura y funcionamiento existen prolíficos armazones teóricos que en muchos casos esclarecen el asunto, pero otros se ubican en el sentido contrario. A continuación, se 
presenta una aproximación al concepto de cultura y sus componentes, denotando los asuntos éticos como uno de ellos. Dado que esta tarea tiene acá un carácter meramente enunciativo, no se pretende agotar la discusión, sino más bien crear un lugar de diálogo para establecer las relaciones más posibles entre la cultura, sus atributos y, específicamente, los sistemas éticos que la componen.

En consecuencia, no es posible pensar la cultura sin los sistemas éticos; son estos los que, mediante sus declaraciones y enunciados, determinan, en cierta medida, la peculiaridad de un sistema cultural. Se empieza, entonces, por hablar sobre la cultura desde su concepto y tradición. Allí un asunto transversal se refiere al objeto de estudio, o aquellos procesos que pretende estudiar. En este sentido, se miran las diferentes aproximaciones históricas que ha tenido el concepto. Cada uno de ellos tiene una impronta particular que, desde una visión global, fue circulada en ambientes geográficos, útiles, pero también legitimados por las necesidades explicativas y/o comprensivas de su momento específico.

Estas ideas, sobre la interpretación del concepto de cultura, atienden un poco la discusión sobre la naturaleza de lo que pretende estudiar, de lo que llamamos acá, como ya lo dijimos, objeto. Sin embargo, no se trata de una visión epistémica centrada solo en las relaciones de este con los objetos que se han delimitado, es, más bien, una mirada sobre cómo las diferentes audiencias, en su momento, interpretaron la cultura.

Ligado a ello, el debate incesante sobre la caracterización de la cultura como concepto resulta emparentado también con los métodos que utiliza para construir conocimiento. Estas formas metodológicas tienen sentido en la medida en que puntúan o definen un objeto de análisis. Las consideraciones respecto de este tema se hacen en menor medida, y solo sirven para mostrar la amplitud de aspectos relacionados con el concepto. Elemento, como ya dijimos, emparentado al objeto de estudio. En suma, son de relieve los diferentes objetos y, en segundo lugar, pero relacionado, sus métodos.

En adición, se enuncian algunos aspectos sobre las relaciones entre la cultura y los conceptos de interculturalidad, sociedad y politica. En su mirada integral, estos elementos hacen posible la comprensión actual de su debate, siempre en arreglo de poder establecer vínculos con la teoría de la moral, aspecto relevante dentro del presente apartado. 


\section{Las miradas al objeto de estudio de la cultura}

La literatura científica del siglo XX, y en gran parte la del naciente siglo XXI, ha registrado aportes significativos en la sistematización del objeto de estudio de la cultura. En el interesante trabajo denominado Ideología y cultura moderna, el británico John Thompson (1998) indica que, dentro del discurso de las ciencias sociales, la cultura se refiere a las formas simbólicas en su relación con la estructura social que les da sentido. Es decir, al estudio del mundo sociohistórico en cuanto campo significativo. Esta perspectiva enriquece el debate en la medida en que se refiere a las formas simbólicas como carácter de la cultura y cómo estas tienen significado a través de los denominados campos. Esta organizada enunciación se robustece mediante el análisis preliminar en torno a los sentidos que ha logrado tener la cultura, en diferentes momentos y desde diversos intereses.

Referirnos entonces desde la perspectiva de Thompson (1988) a los sentidos atribuidos a la cultura, implica admitir que se pueden trazar de manera independiente por lo menos cuatro comprensiones posibles, las cuales se distinguen por la forma en la cual se ha hecho presente el concepto de cultura en el debate académico (tabla 1).

Tabla 1. Propuesta de perspectivas en torno al concepto de cultura

\begin{tabular}{|c|c|c|}
\hline & Sentidos & Características \\
\hline 1 & $\begin{array}{l}\text { Concepción clásica de la cultura } \\
\text { (bumanista) }\end{array}$ & $\begin{array}{l}\text { Filósofos e historiadores alemanes durante los } \\
\text { siglos XVIII y XIX. Desarrollo intelectual y espi- } \\
\text { ritual diferente al concepto de civilización. }\end{array}$ \\
\hline 2 & $\begin{array}{l}\text { Concepción descriptiva } \\
\text { Antropología a finales del siglo } \\
\text { XIX }\end{array}$ & $\begin{array}{l}\text { Aspectos integrales de la cultura. Sistemas } \\
\text { éticos, creencias, costumbres, convenciones, } \\
\text { hábitos y prácticas; sociedad en periodo histó- } \\
\text { rico (Tylor, 1871; Malinowski, 1944). }\end{array}$ \\
\hline 3 & $\begin{array}{l}\text { Concepción simbólica } \\
\text { Antropología a finales del siglo XX }\end{array}$ & $\begin{array}{l}\text { Interpretación de los símbolos y acción simbó- } \\
\text { lica (Geertz, 1973). }\end{array}$ \\
\hline 4 & $\begin{array}{l}\text { Concepción estructural } \\
\text { (Thompson) }\end{array}$ & $\begin{array}{l}\text { El análisis de la cultura es el estudio de la cons- } \\
\text { trucción significativa y de la contextualización } \\
\text { social de las formas simbólicas (Thompson, } \\
\text { 1998). }\end{array}$ \\
\hline
\end{tabular}

Fuente: elaboración propia. 


\section{Mirada "clásica" de la cultura, lo humanista}

El primer sentido se refiere a la interpretación que los filósofos alemanes e historiadores, particularmente de los siglos XIX y XX, le atribuyeron a la cultura. Dada la aceptación del concepto dentro de los ambientes y las audiencias académicas de su tiempo, este logró tener adhesión en la medida en que podría entenderse como desarrollo intelectual o espiritual y que tenía diferencia con el concepto de civilización. No obstante, antes de continuar es menester indicar algunas ideas sobre las definiciones y sentidos de la palabra:

De la palabra culturam, el concepto adquirió una presencia significativa en muchas lenguas europeas durante el inicio del periodo moderno. Los primeros usos dados al concepto en las lenguas europeas preservaron parte del sentido original de cultura, el cual significaba primordialmente el cultivo o el cuidado de algo, como las cosechas o los animales. A partir del siglo XVI, el sentido original se extendió poco a poco de la esfera de la labranza al proceso del desarrollo humano: pasó del cultivo de las cosechas al cultivo de la mente. Sin embargo, el uso del sustantivo independiente cultura, para referirse a un proceso general o al producto de dicho proceso, no fue algo común sino hasta finales del siglo XVIII y comienzos del XIX. El sustantivo independiente apareció primero en francés e inglés; y a finales del siglo XVIII, la palabra francesa se incorporó al alemán, en el que primero se escribía Cultur y más tarde Kultur. (Thompson, 1988, p. 3)

La primera concepción que Thompson le atribuye a la cultura, como ya lo hemos indicado, se refiere al uso que le dieron los historiadores alemanes pertenecientes al mundo intelectual, y en menor medida al cortesano y burgués, asociándolo al concepto de desarrollo de las facultades intelectuales, en contraposición con la forma que otro grupo social, en menor medida intelectual y más cercano al uso de la lengua francesa, le daba emparentándolo al concepto de civilización. Desde esta perspectiva se asoció al refinamiento de los modales que se usaban en la vida de las cortes y los cortesanos.

Los alemanes de finales del siglo XVIII y principios del siglo XIX, que usaron el concepto como el proceso mediante el cual los sujetos adquieren conocimiento y perfeccionan sus capacidades mentales, en el marco amplio de la ciencia y sus desarrollos, fueron contrarios a la concepción atribuida al refinamiento de los modales. Esto se hizo evidente en las notables obras que en materia de historia se formularon. 
Los textos que narraban los sucesos del mundo con frecuencia describían el proceso de ennoblecimiento de la condición humana, en términos análogos al desarrollo de la cultura. Esta posición se afincaba fundamentalmente en la apuesta que la humanidad le daba, en el siglo XVIII, a la razón y a su proyecto de desarrollo. Aquel panorama fue enriquecido por una atmósfera plagada de ilustración, que conectaba las puntuaciones de la historia de la humanidad con la lectura de textos clásicos que, muy seguramente, dejaban de lado la posibilidad de la observancia de otros valores diferentes, que no formaban parte del ámbito europeo, pero que sí constituían otras posibilidades expresivas de la humanidad.

$\mathrm{Al}$ respecto, y en perspectiva de pensar algunos avances y transformaciones a esta mirada humanística, pero también de darles tránsito a los nuevos conceptos que la naciente ciencia antropológica traía, Thompson (1998) concluye que progresivamente se logró despojar al concepto de cultura de algunas de sus connotaciones etnocéntricas, principalmente europeas, y se adaptó a las tareas de la descripción etnográfica.

El estudio de la cultura trataba ahora menos del ennoblecimiento de la mente y el espíritu en el corazón de Europa, y se interesaba más por descifrar las costumbres, prácticas y creencias de aquellas sociedades que constituían el otro para Europa. (Thompson, 1988, p. 6).

\section{Miradas antropológicas. Lo descriptivo y lo simbólico}

La segunda concepción de Thompson (1988) muestra su interés, dentro del seno de la naciente ciencia antropológica a principios del siglo XIX, por dos maneras de entender la cultura. La primera es la perspectiva descriptiva, y la segunda es la perspectiva simbólica. Aunque sean dos miradas diferentes, Thompson las agrupa en el mismo eje disciplinar.

La perspectiva descriptiva, sobre la cual haremos consideraciones primero, pretendió apreciar las sociedades no europeas del siglo XIX. Aquella mirada no se centraba en la comprensión literaria o humanística como supuesto de la cultura; por el contrario, se interesó por hacer de los componentes de la cultura unidades observables bajo el lente de la ciencia. Al respecto, Taylor (1871), profesor de antropología de la Universidad de Oxford, en su eminente obra Cultura primitiva, indica: 
La cultura o civilización, tomada en su sentido etnográfico amplio, es esa totalidad compleja que abarca el conocimiento, las creencias, el arte, la moral, la ley, las costumbres y cualesquiera otras habilidades y hábitos adquiridos por el hombre como miembro de la sociedad. La condición de la cultura entre las diversas sociedades de la humanidad, en la medida en que se puede investigar a partir de principios generales, es un tema propicio para el estudio de las leyes del pensamiento y la acción humanas. (Taylor, 1871, p. 1)

La perspectiva descriptiva de la cultura notablemente tomó distancia de la concepción de un asunto centrado en el ennoblecimiento de las cualidades humanas, en tanto lo humano lograba mayores niveles de desarrollo y evolución; concepción esta dentro del marco de la ciencia histórica.

Se dirigió de manera diferente al análisis científico y la cientificidad del concepto mediante la comparación, la clasificación y la descomposición de los elementos constitutivos de diversas culturas; ello también como la perspectiva clásica de la ciencia alemana, sobre un presupuesto evolutivo que discurre desde el salvajismo hasta las formas civilizadas. Estas ideas tuvieron un clima favorable durante el siglo XIX y permitieron, junto a la consolidación de las ciencias positivas y las ideas de Darwin, alcanzar preeminencia dentro de la sociedad académica.

De la década de 1930 a 1940, la teoría científica de la cultura tuvo acogida dentro de la propuesta de Malinowski, quien, desde un enfoque funcionalista entendió que los fenómenos culturales se pueden estudiar en términos de la satisfacción de las necesidades humanas específicas y concibió como responsable de esta tarea a la antropología cultural. Al respecto de la conjunta mirada Taylor-Malinowski (descriptiva), Thompson (1998) indica:

La cultura de un grupo o sociedad es el conjunto de creencias, costumbres, ideas y valores, así como los artefactos, objetos e instrumentos materiales que adquieren los individuos como miembros de ese grupo o esa sociedad; agrega que el estudio de la cultura implica, al menos en parte, el análisis, la clasificación y la comparación científicas de estos diferentes fenómenos. (Thompson, 1998, p. 9)

Desde el punto de vista descriptivo del análisis cultural, propio del estadio primero de la antropología, la cultura se pretende objeto de estudio de esta ciencia. Para ello intenta definir su sentido y sus características. Sin embargo y 
en la perspectiva de Thompson, no se hace un abordaje directo a los sistemas metodológicos propios de la investigación social que permitan asir este objeto.

La perspectiva descriptiva le otorgó a la cultura un lugar protagónico en el engranaje de la ciencia social. La identificó y la ubicó como objeto posible de ser descrito dentro de los lineamientos de la ciencia empírica. Este hecho, sin lugar a dudas, le permitió a la cultura hacer el tránsito de la concepción humanista a la concepción cientificista. Describió con cierta sistematicidad los componentes o atributos de la cultura, y trazó el camino para que las ulteriores generaciones de científicos sociales pudieran tener en este concepto un lugar donde trabajar y descubrir.

Sin embargo, con el advenimiento de nuevos modelos de ciencia, y la proliferación de teorías comprensivas del mundo, en la medida en que iba robusteciéndose lo descriptivo de la cultura, también floreció un análisis simbólico preocupado por los sentidos de esta, ya no por sus clasificaciones y mediciones con corte cientificista. En toda la concepción simbólica, existe la intención de referirse a un modelo diferente de entender la cultura. Camina sobre los senderos de la comprensión y abandona, en cierta medida, los de la explicación.

Al respecto, en su obra Filosofía de las ciencias humanas y sociales, Mardones (2001) hace un análisis sobre las diferentes racionalidades de las ciencias de la naturaleza y las ciencias del espíritu. Describe cómo estos modelos se constituyeron con anterioridad, para luego convertirse en las clasificaciones actuales de la ciencia:

Desde finales del siglo XIX estalló la polémica entre ciencias de la naturaleza y ciencias del espíritu. Dilthey, alumno de Ranke, quiere fundamentar la historia y demás ciencias que se relacionan con el hombre en cuanto ser histórico y social. Descubre que la teoría del conocimiento de las ciencias naturales no hace justicia a su peculiaridad. Por esta razón, tratará de fundamentar lo que denomina "ciencias del espíritu". El objeto de estas ciencias no es lo externo o ajeno al hombre, sino el medio en el que el hombre está inserto. Este hecho hace que el hombre pueda captar su mundo histórico social como desde dentro. De esta singularidad de relaciones entre el sujeto y el objeto de las ciencias del espíritu, frente a las ciencias naturales, deducirá Dilthey su diferente metodología. (Mardones, 2001, p. 87) 
La indicación de Mardones (2001) sobre los planteamientos de Dilthey resulta fecunda en la medida en que posiblemente en la antropología se presentó esta doble mirada paradigmática. Por un lado, la descriptiva cientificista, remembrando la racionalidad propia de las ciencias naturales, y la simbólica por el otro, atendiendo la racionalidad de las ciencias del espíritu, que ubican al hombre como ser histórico y social suspendido en tramas de significación tejidas por él mismo. En consecuencia, la perspectiva simbólica del análisis cultural se centró en la aceptación de que el hombre y su expresión no pueden comprenderse solo por los métodos trazados por el rigor de la ciencia positiva.

Los trabajos de L. A. White en Science of culture y Clifford Geertz en The interpretation of cultures (1973) son puntos de partida para la explanación de la simbólica en la antropología. Al respecto, Geertz (1973) señala que la cultura es el patrón de significados incorporados a las formas simbólicas —entre las cuales se incluyen acciones, enunciados y objetos significativos de diversos tiposen virtud de los cuales los individuos se comunican entre sí y comparten sus experiencias, concepciones y creencias.

El análisis cultural es fundamentalmente la enunciación de los patrones de significado. Es una acción dentro del marco general de la interpretación, en eso, incluso semejante a la de un texto. En contraposición a la perspectiva cientificista de la antropología, cuyo interés se centró en la clasificación y jerarquización, más que en el ejercicio interpretativo.

Sin embargo, y a pesar de que Geertz (1973) constituye un punto de referencia para la simbólica en la cultura, el estudio de sus postulados le permitió a Thompson (1998) indicar algunas dificultades:

Geertz ofrece la formulación más importante del concepto de cultura que ha surgido de la literatura antropológica. Ha reorientado el análisis de la cultura hacia el estudio del significado y del simbolismo, y ha puesto de relieve la centralidad de la interpretación como enfoque metodológico. No obstante, en su trazado investigativo: a) aun cuando se intenta formular una caracterización precisa de la concepción simbólica, usa el término cultura de varias maneras diferentes, de las cuales no todas parecen ser totalmente consistentes; b) tampoco establece con precisión el significado de la unidad textual (texto), sus características y objeto de análisis metodológico, y c), por último, no presta 
suficiente atención a los problemas del poder y el conflicto social. (Thompson, 1998, pp. 12-15)

\section{Mirada estructural de la cultura}

La concepción estructural de la cultura acepta los postulados de la mirada simbólica y los componentes de significado relacionados. Esto implica el énfasis en la comprensión, más que en la explicación. De manera general, para Thompson (1998), el objeto del análisis cultural se centra en la apreciación de las formas simbólicas, que, entre otras, son las manifestaciones de la atmósfera y el cuerpo de la cultura; en ellas se expresan los componentes o atributos de la particularidad de ser de un colectivo, enquistado sociohistóricamente. El trazado más básico de esta postura, como ya dijimos, es que admite el carácter simbólico del concepto, pero añade que estas formas simbólicas se instauran en procesos socialmente estructurados.

Representa entonces una postura que integra lo simbólico a la estructura social. Para ello recurre a la explicación comunicativa, evidente en las denominadas características de las formas simbólicas, que son componentes esenciales para la comprensión de la cultura. Las formas simbólicas se insertan en estructuras socialmente estables. Sobre lo anterior, Thompson (1998) señala que,

como formas simbólicas, los fenómenos culturales son significativos tanto para los actores como para los analistas. Son fenómenos que los actores interpretan de manera rutinaria en el curso de sus vidas diarias y que reclaman una interpretación por parte de los analistas que buscan captar las características significativas de la vida social. No obstante, estas formas simbólicas se insertan en contextos y procesos sociohistóricos en los cuales, y por medio de los cuales, se producen, transmiten y reciben. (p. 16)

En coherencia, es posible agregar que el contenido simbólico se expresa de variadas maneras, entre las cuales podrían ponerse de relieve las representaciones sociales, que son parte del mundo de la interacción:

La teoría se refiere a la vinculación que tenemos en el mundo social en cuanto compartimos modos de pensar y actuar. Es, entonces, una propuesta de pensamiento social, una forma de explicar los lazos que nos unen tácitamente y que, en gran medida, matizan nuestras acciones cotidianas. En ese sentido, no es posible referirse a una representación en solitario, será menester el otro. Es con 
él y mediante ello como se construyen, transmutan y establecen los dominios del pensamiento social. (Pérez et al., 2012, p. 131)

\section{Conclusión}

\section{Sobre la cultura de la formación ética}

La intención más elemental de esta reflexión, como ya se indicó en apartados anteriores, fue, a partir de los conceptos elegidos en Kant y la teórica de la cultura, proponer como punto de partida algunos elementos indispensables al momento de pensar la formación ética. En consecuencia, la estructura propuesta debe incluir lo señalado en el primer apartado de este artículo, expresado en los tres aspectos subrayados en la Fundamentación de una metafisica de las costumbres: la concepción del hombre como finalidad en sí mismo, valor absoluto e independiente de instrumentalización alguna, el reconocimiento de los imperativos categóricos y el afianzamiento de la voluntad y su cualidad autónoma.

Asimismo, y desde la aportación proveniente de la teorética de la cultura, proponer que el enfoque simbólico constituye la mirada más amplia en donde los principios y aspectos kantianos podrían converger. En adición, pensar estos elementos en conjunto implica centrarnos en el aparato educativo, para, desde él, desentrañar la aplicabilidad de lo mencionado.

Desde el punto de vista de la estructura que permite poner en funcionamiento los idearios educativos expuestos, es menester recordar que su inclusión debe hacerse evidente en todos los niveles de la institución educativa. Así, sería necesario pensar cómo es posible direccionar una ética educativa que atraviese los diseńos micro, meso y macrocurriculares. Desde este último, es deseable que la estrategia organizacional pensada como propuesta educativa incluya las consideraciones éticas expuestas a lo largo del escrito. Desde lo mesocurricular, es sugerente adoptar medidas que puedan atravesar los distintos organismos académicos que se agrupan habitualmente en torno a saberes específicos. Por último, en el nivel microcurricular se expresa en la inclusión de principios éticos centrados en la mirada kantiana, y cuya disposición atraviesa los programas y propuestas específicas como planes de formación. Se trata entonces de una mirada integral total de toda la organización 
educativa, volcada a pensar la formación ética sobre idearios humanos que reafiancen la dignidad de la persona en cuanto valor absoluto, la construcción de la autonomía de la voluntad y el reconocimiento de las leyes éticas compartidas universalmente.

En adición, y contrario a lo que podría pensarse respecto de las audiencias que reflexionan en torno a aspectos éticos, el espíritu del proceso formativo debe incluir todos los niveles de formación. Así, se hace necesario que desde el nivel preescolar se establezcan comunidades de diálogo filosófico, con preguntas coherentes a los sistemas de desarrollo cognitivo específicos y que provean reflexiones y conclusiones éticas al servicio del buen vivir. Por supuesto, esta formación debe irradiarse incrementalmente en la formación profesional, pues es allí donde la conducta ética tiene un nicho específico de realización, concreción y relevancia social.

Los aprendizajes derivados de una propuesta ética actual deben ser dinamizados por colectivos entrenados en pensamiento y reflexión sobre asuntos morales y, consecuentemente, culturales. En el aula, "de esta manera, la práctica pedagógica es una práctica discursiva inscrita en el saber de la educación, que entre sus múltiples objetos destaca el otorgamiento de sentido a lo que dice y hace el maestro" (Pérez et al., 2013, p. 112).

La ciencia militar, en cuanto actualización y sistematización de su objeto y producción científica, fortalecerá la formación ética como un proceso gradual en todos los niveles de formación, que parta de la problematización y el reconocimiento de las discusiones más actuales del proceso ético, hasta lograr competencias afianzadas en la comprensión de un sujeto fin en sí mismo, con uso razonable de su voluntad y autonomía concomitantes.

\section{Referencias}

Bauman, Z. (2010). La cultura como concepto. En La cultura como praxis (pp. 95-175). Paidós.

Kant, I. (1785). Fundamentación de una metafísica de las costumbres [Versión castellana y estudio preliminar de Roberto R. Aramayo]. Alianza.

Geertz, C. (1973). The interpretation of cultures. Basic Books.

Malinowski, B. (1944). A scientific theory of culture and other essays. University of North Carolina Press. 
Mardones, J. M. (2001). Filosofía de las ciencias humanas y sociales. Materiales para una fundamentación cientifica. Anthropos.

Pérez, L., Arenas, M., \& López, S. (2013). Prácticas docentes y expectativas de los empleadores. Una mirada a sus relaciones en perspectiva de la Educación Física. Lúdica Pedagógica, 2(18), 111-122. https://doi.org/10.17227/

Pérez, L., Arenas, M., \& López, S. (2012). Representaciones sociales que tienen los docentes del programa de Educación Física de la Universidad Pedagógica Nacional sobre las competencias propias de su saber disciplinar. Lúdica Pedagógica, 2(17), 130-137. https://doi. $\operatorname{org} / 10.17227 /$

Rodríguez, L., \& Aguirre, J. (2011). Teorías de la complejidad y ciencias sociales. Nuevas estrategias epistemológicas y metodológicas. Nómadas. Critical Journal of Social and Juridical Sciences, 30(2). https://www.redalyc.org/pdf/181/18120143010.pdf

Thompson, J. B. (1998). El concepto de cultura. En Ideología y cultura moderna. Teoría crítica social en la era de la comunicación de masas (pp. 183-240). Casa Abierta al Tiempo.

Tylor, E. (1871). Primitive culture: Researches into the development of mythology, philosophy, religion, language, art and custom (vol. 1). John Murray. 